U. of Iowa 91-19

\title{
New ObServations of THE Low Frequency INTERPLANETARY RADIO EMISSIONS
}

\author{
W. S. Kurth and D. A. Gurnett
}

August 1991

Submitted for publication to Geophysical Research Letters.

\author{
Department of Physics and Astronomy \\ The University of Iowa \\ Iowa City, IA 52242
}

This research was supported by the National Aeronautics and Space Administration through Contract 957723 with the Jet Propulsion Laboratory. 
$=$ 
Submitted for publication in Geophysical Research Letters.

U. of Iowa 91-19

\title{
NEW OBSERVATIONS OF THE LOW FREQUENCY INTERPLANETARY RADIO EMISSIONS
}

\author{
W. S. Kurth and D. A. Gurnett \\ Department of Physics and Astronomy, The University of Iowa, Iowa City
}

\begin{abstract}
Recent Voyager 1 observations reveal reoccurrences of the low frequency interplanetary radio emissions. Three of the new events are weak transient events which rise in frequency from the range of $2-2.5$ $\mathrm{kHz}$ to about $3 \mathrm{kHz}$ with drift rates of $\sim 1.5 \mathrm{kHz} /$ year. The first of the transient events begins in mid-1989 and the more recent pair of events both were first detected in late 1991. In addition, there is an apparent onset of a 2$\mathrm{kHz}$ component of the emission beginning near day 70 of 1991. The new transient emissions are barely detectable on Voyager 1 and are below the threshold of detectability on Voyager 2, which is less sensitive than Voyager 1. The new activity provides new opportunities to test various theories of the triggering, generation, and propagation of the outer heliospheric radio emissions and may signal a response of the source of the radio emissions to the increased solar activity associated with the recent peak in the solar cycle.
\end{abstract}

\section{INTRODUCTION}

As early as 1983 the plasma wave receivers on the Voyager 1 and 2 spacecraft began detecting radio emissions in the frequency range of $2-3.5 \mathrm{kHz}$ [Kurth et al., 1984a, 1987]. These very weak emissions generally appeared in two bands, one near $2 \mathrm{kHz}$ which was visible nearly continuously until about 1987 and another, more transient component near $3 \mathrm{kHz}$ which showed upward frequency drifts of about $1 \mathrm{kHz} /$ year in two primary events, one beginning in late 1983 and the other in late 1985. These two higher frequency bursts lasted for $\sim 9$ months. While we have taken precautionary statements about the interpretation of these events seriously [c.f. Meyer-Vernet, 1989], considerable evidence exists to show that these emissions are freely-propagating radio emissions [Kurth et al., 1984a; Kurth, 1990; Kurth and Gurnett, 1991]. The best current explanation proposed for the source of these emissions is the conversion of Langmuir waves into electromagnetic radiation at the plasma frequency $f_{p}$ or its second harmonic. The Langmuir wave generation is believed to be associated with the heliospheric termination shock at $\sim 50$ to 100 AU.

Recently, a number of theoretical advances have been made on the triggering, generation, and propagation of the low frequency radio emissions. First, McNutt [1988] has suggested that very high speed solar wind streams may trigger the emissions when the streams reach the source region, which is presumed to be the termination shock. Unfortunately, the availability of only two events provides a relatively poor test of the triggering hypothesis; the 1983 event seems to have a well-defined trigger, but the case is not as clear for the 1985 event. Second, Macek et al. [1991a,b], Cairns and Gurnett [1991], and Caims et al. [1991] have carried out the first quantitative evaluations of a source mechanism involving the nonlinear decay of Langmuir waves upstream of the termination shock into electromagnetic radiation at $f_{p}$ and $2 f_{p}$. These studies, while not proving the termination shock is the source of the low frequency radiation, show the feasibility of the mechanism. Third, Czechowski and Grzedzielski [1990] offered an explanation for the upward frequency drift of the emissions, based primarily on the Fermi scattering of the waves off of density structures moving outward from the sun at usual solar wind velocities. This process results in a net increase in frequency after multiple reflections; the theory assumes that the waves are trapped within the heliospheric cavity by a relatively dense plasma surrounding it.

All of these theoretical works would benefit by additional observations to further test and constrain the theories. In this paper we report three additional transient events extending up to $\sim 3 \mathrm{kHz}$ and a possible new onset of the 2-kHz component, all observed by Voyager 1 since 1989.

\section{ObSERVATIONS}

The primary method of detecting the very weak low frequency radio emissions is by averaging over 15-s intervals of wideband observations taken about once per week by both Voyager plasma wave instruments. The wideband portion of the plasma wave receiver is more serisitive when used in this way than the spectrum analyzer portion of the instrument, especially on Voyager 2. By taking the averaged spectra and stacking them side-byside, we construct long-period frequency-time spectrograms which show the temporal evolution of the wave spectrum [Kurth et al., 1987]. Figure 1 shows the Voyager 1 wideband data in the frequency range of 1.5 to $4 \mathrm{kHz}$ for the period 1983 through day 122 of 1991 . Here, the ordinate is frequency, the abscissa is time, and a gray scale is used to indicate relative wave intensity wherein black is the most intense and white the least. A severe stretch of the amplitude range has been used to highlight the low frequency emissions. The peak of the $19833-\mathrm{kHz}$ event has a power flux of about $3 \times 10^{-17}$ 


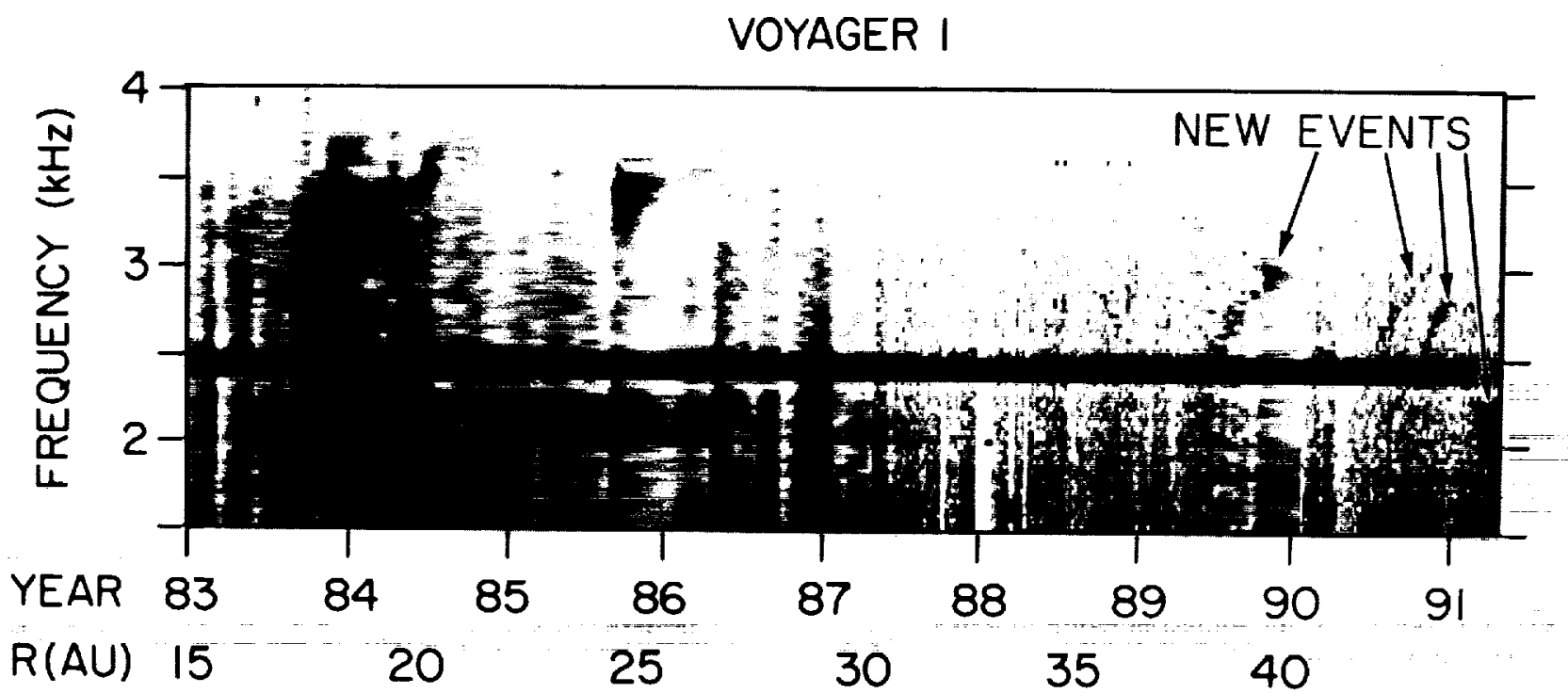

Figure 1. A frequency-time spectrogram constructed from Voyager 1 wideband observations spanning the interval from the beginning of 1983 through May 2, 1991. The low frequency interplanetary radio emissions are clearly present in this display. The transient events near $3 \mathrm{kHz}$ beginning in late 1983 and late 1985 and the more continuous $2-\mathrm{kHz}$ component appearing through mid-1987 have all been reported previously. The new observations presented herein are the three transient signals rising in frequency in the range of 2 to $3 \mathrm{kHz}$ in 1989 and 1990 and the apparent reappearance of the $2-\mathrm{kHz}$ component in 1991.

$\mathrm{W} / \mathrm{m}^{2} \mathrm{~Hz}$. The noise level is only a factor of two less than this.

The newly observed events appear in Figure 1 beginning about mid-1989 through the end of 1990 . The events appear as weak, narrowband rising tones beginning in the range of $2-2.5 \mathrm{kHz}$ and ending near $3 \mathrm{kHz}$. An intensification in the $2-\mathrm{kHz}$ range can be seen beginning on about day 71 of 1991 . The three transient events are weaker than the previously reported events, but these plus the recent $2-\mathrm{kHz}$ intensification mark an onset of low frequency radio wave activity after a period of about 3 years with little or no positive detection.

We emphasize the very weak nature of the new transient events. Frankly, the emissions do not show a significant peak in the spectrum during many of the weekly samples and it is only by noticing the coherent coalescence of those peaks into a recognizable feature in the frequency-time spectrogram which makes the identification possible. This method of identification is similar in many respects to finding weak lightning whistlers in the wideband data. Often, the individual spectra show only very weak spectral peaks, but the frequency-time spectrograms show the distinctive temporal evolution [see, for example, Gurnett et al., 1990].

The consequence of the weak nature of the new emissions is that the Voyager 2 data fail to show definitive evidence of the three transient events. As discussed in Kurth et al. [1984b], the Voyager 2 receiver is a couple $\mathrm{dB}$ less sensitive than that on Voyager 1 . This can be seen by noting the differing noise levels between the two sets of observations in Figure 4 of that paper.

In the past, we have taken great comfort in observing these emissions on both spacecraft as evidence of a nonlocal phenomenon. We have tried to stretch the Voyager 2 spectrogram's intensity scale in order to bring any evidence of these features to light. It is possible to find apparent intensifications at the proper frequencies and times and there is even a suggestion of the drifting structures in the Voyager 2 data. However, we do not think we could have independently found these events with the Voyager 2 data.

In spite of a lack of confirmation from Voyager 2, the Voyager 1 observations appear to be real, natural signals and are similar in spectral form to the $19833-\mathrm{kHz}$ event so that we feel confident in identifying them as the same phenomenon. While it is more difficult to rule out local effects [Meyer-Vernet, 1989] without complementary Voyager 2 observations, we believe the cohesive drifting structure which transcends several solar rotations (when the local medium would exhibit quasiperiodic variations) is reason enough to rule out local effects.

Because the above-mentioned theoretical work is concemed with correlating solar wind events with the onset of emissions to establish a triggering mechanism and also to understand the drifting frequency of the emissions, we present the new observations on an expanded time scale in Figure 2, covering the period from the beginning of 1989 through day 122 (May 2) of 1991. Figure 2 allows a more accurate determination of both the slope of the transient events and the onset dates. 
VOYAGER I

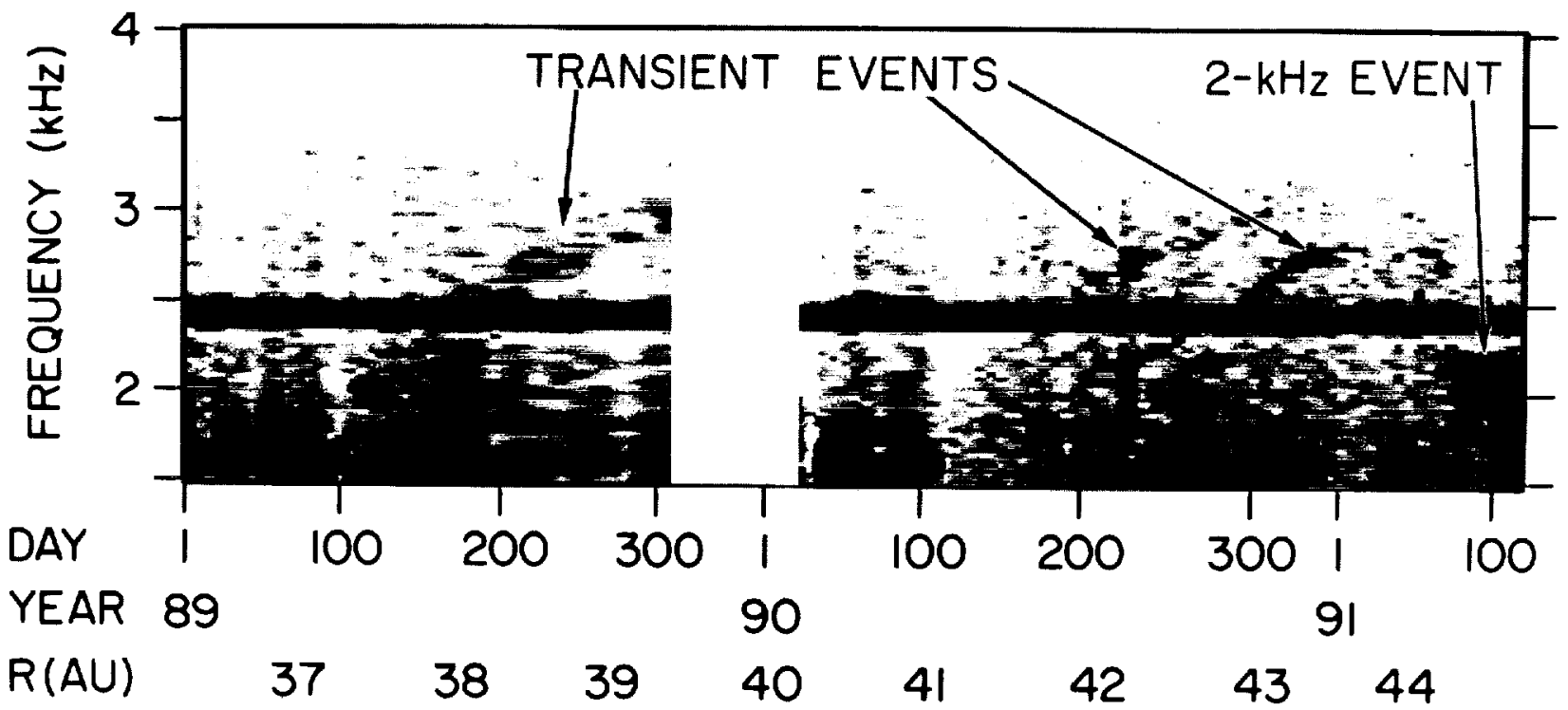

Figure 2. An expansion of the data in the previous figure for the period from 1989 through early 1991 showing the newly observed emissions. The expanded scale allows for more accurate determination of onset times and frequency drift rates for comparison with theory.

The weak nature of the events makes it rather difficult to accurately determine the onset times. First, regardless of the intensity, these measurements are only available every week or so. Hence, there is up to a 7-day inaccuracy. Second, finding the spectrum with the first evidence of emission is difficult because of the very weak signal strength. Third, the presence of the $2.4-\mathrm{kHz}$ interference tone and the notch filter in the receiver centered on that frequency [Scarf and Gurnett, 1977] makes the onset time even more susceptible to error. All of these errors lead to an identification of onset time which is probably later than the actual one.

Table 1 provides the onset time, beginning frequency, ending frequency, and drift rate for the three new transient events. One might notice a possible inflection or change in slope in the last transient event. This occurs as the band passes through the region of the notch filter and 2.4$\mathrm{kHz}$ interference tone, hence, we are reluctant to identify the inflection as a true signature. On the other hand, the 1989 event appears to pass through the notch with no apparent inflection (or perhaps one with a reverse sign).

Finally, the available Voyager 2 data set does not include data beyond day 72 of 1991, hence, we cannot judge whether the $2-\mathrm{kHz}$ event is visible from Voyager 2 or not, yet. It appears that the new Voyager $12-\mathrm{kHz}$ event is somewhat different from simply another transient event which would eventually drift higher in frequency. It is too early to speculate on how this most recent signature might evolve.

\section{Discussion}

The observations presented herein are of great importance to the ongoing theoretical work mentioned in the introduction. Certainly, it is now possible to test the triggering hypothesis with more events. While McNutt [1988] was only able to identify a trigger for the first of the two previously identified transient $(3-\mathrm{kHz})$ events, Grzedzielski and Iazarus [personal communication, 1991] and Grzedzielski [1991] have refined somewhat the method of identifying triggers by finding groups of fast streams which tend to merge at or near the source region following general increases in the ram pressure in the outer heliosphere. They claim triggering events for both of the earlier events as well as the 1989 event shown here. In turn, a successful triggering hypothesis will provide a distance to the source based on the transit time of the trigger [McNutt, 1988].

The modeling of frequency drift will need to address the somewhat more rapid frequency drift rates $(\sim 1.5$ $\mathrm{kHz} /$ year) of the recent events. Faster drift rates would seem to imply a smaller scale size for the heliospheric cavity but the modeling is currently being advanced to take into account such features as corotating interaction regions [S. Grzedzielski, personal communication, 1991] so such an extrapolation may be premature.

The appearance of both the transient events and now, perhaps, the steadier $2-\mathrm{kHz}$ component will further constrain the modeling of the generation mechanism [Caims et al., 1991]. This effort attempts to sort out the different emissions as being due to different mechanisms (e.g. $f_{p}$ or $2 f_{p}$ emission) or different source regions (e.g. different solar latitudes) or both.

One conclusion may be drawn from the transient events observed to date on the assumption that the Czechowski and Grzedzielski [1990] Fermi scattering model is the 
Table 1

\begin{tabular}{cccc}
\hline $\begin{array}{c}\text { Start Date } \\
\text { (Year/Day) }\end{array}$ & $\begin{array}{c}\text { Starting Frequency } \\
(\mathrm{kHz})\end{array}$ & $\begin{array}{c}\text { Ending Frequency } \\
(\mathrm{kHz})\end{array}$ & $\begin{array}{c}\text { Drift Rate } \\
(\mathrm{kHz} / \text { year })\end{array}$ \\
\hline \hline $1989 / 155$ & 2.0 & 3.1 & $1.4^{*}$ \\
$1990 / 205$ & 2.5 & 3.2 & 1.4 \\
$1990 / 215$ & 2.1 & 2.8 & 1.8 \\
\hline \hline
\end{tabular}

*Measured for the portion of the event above $2.5 \mathrm{kHz}$.

appropriate explanation for the frequency drift. Since this model depends on trapping, it implies that the plasma surrounding the heliospheric cavity has a plasma frequency in the range of 3 to $3.5 \mathrm{kHz}$ based on the maximum frequencies of the up-shifted waves. Hence, the surrounding plasma density must be in the range of 0.1 to $0.15 \mathrm{~cm}^{-3}$. If we assume the boundary of the cavity is the very local interstellar medium (VLISM), this result is, on the surface, in conflict with the results of LISM observations using nearby stars [Frisch, 1990]. The stellar observations suggest a neutral density of about 0.1 $\mathrm{cm}^{-3}$. Given that the temperature of the LISM neutral gas is approximately $10^{4} \mathrm{~K}$ [Frisch, 1990], the degree of ionization is commonly expected to be of the order of $10 \%$, hence, the plasma densities obtained from the stellar observations are roughly an order of magnitude too low to be consistent with the low frequency radio observations. Obviously, the stellar observations integrate over a great distance, hence, perhaps the solution to this dilemma is that the VLISM is more dense than the average, including the surrounding medium out to several parsecs.

The increase in low frequency radio emission activity in the 1989-1991 time frame following a dearth of activity over the preceding few years suggests a solar cycle variation to the activity. The initial activity was seen in 1983 with the most intense event observed in the declining phase of the solar cycle. At this time, however, the Voyager spacecraft had only recently entered a regime in the solar wind where the local plasma frequency was low enough to allow the observation of these emissions. If this pattern of activity continues, it provides even more evidence of triggering by solar events. If increased solar activity is responsible for radio bursts which exceed the Voyager detection threshold, then we should expect continued activity through the declining phase of the current solar cycle.

Acknowledgements. This research was supported by the National Aeronautics and Space Administration through Contract 957723 with the Jet Propulsion Laboratory.

\section{REFERENCES}

Cairns, I. H., and D. A. Gurnett, The outer heliospheric radio emissions: (1) Constraints on emission processes and the source region, J. Geophys. Res., submitted, 1991.
Cairns, I. H., W. S. Kurth, and D. A. Gumett, The outer heliospheric radio emissions: (2) Foreshock source models, $J$. Geophys. Res., submitted, 1991.

Czechowski, A., and S. Grzedzielski, Frequency drift of $3-\mathrm{kHz}$ interplanetary radio emissions: Evidence of Fermi accelerated trapped radiation in a small heliosphere? Nature, 344, 640, 1990.

Grzedzielski, S., Conflicting observational evidence on the confinement of the heliosphere, presented at the IUGG-IAGA meeting in Vienna, Austria, 11-24 August, 1991.

Gurnett, D. A., W. S. Kurth, I. H. Caims, and L. J. Granroth, Whistlers in Neptune's magnetosphere: Evidence of atmospheric lightning, J. Geophys. Res., 95, 20,967, 1990.

Frisch, P. C., Characteristics of the local interstellar medium, in Physics of the Outer Heliosphere, COSPAR Colloquia, vol 1, edited by S. Grzedzielski and D. E. Page, pp 19-28, Pergammon, Oxford, 1990.

Kurth, W. S., Radio noise in the heliospheric cavity, in Physics of the Outer Heliosphere, COSPAR Colloquia, vol 1, edited by S. Grzedzielski and D. E. Page, pp 267-275, Pergammon, Oxford, 1990.

Kurth, W. S., and D. A. Gurnett, Evidence that the Voyager observations of $2-3 \mathrm{kHz}$ radio emissions in the outer heliosphere are not caused by local spacecraft-plasma interactions, Astron. Astrophys., submitted, 1991.

Kurth, W. S., D. A. Gurnett, F. L. Scarf, and R. L. Poynter, Detection of a radio emission at $3 \mathrm{kHz}$ in the outer heliosphere, Nature, 312, 27, $1984 \mathrm{a}$.

Kurth, W. S., D. A. Gurnett, F. L. Scarf, and R. L. Poynter, A new radio emission at $3 \mathrm{kHz}$ in the outer heliosphere, in Proceedings of a Course \& Workshop on Plasma Astrophysics, (ESA SP-207), Varenna, Italy, August 28-September 7, 285, 1984b.

Macek, W. M., I. H. Cairns, W. S. Kurth, and D. A. Gurnett, Plasma wave generation near the inner heliospheric shock, Geophys. Res. Lett., 18, 357, 1991 a.

Macek, W. M., I. H. Cairns, W. S. Kurth, and D. A. Gurnett, Low-frequency radio emissions in the outer heliosphere: Constraints on emission processes, J. Geophys. Res., 96, 3801, 1991 b.

McNutt, R. L., Jr. A solar wind "trigger" for the outer heliospheric radio emissions and the distance to the terminal shock, Geophys. Res. Lett., 15, 1307, 1988.

Meyer-Vernet, N., Electric antennae in the outer heliosphere: the importance of being stable, Astron. Astrophys., 224, L5, 1989.

Scarf, F. L., and D. A. Gurnett, A plasma wave investigation for the Voyager mission, Space Sci. Rev., 21, 289, 1977. 